conferences with the unit nurses about the emotional problems that regularly arise in such places. They were consulted mainly on account of overt anxiety (47 times), depression (44), hostility (12), delirium (11). Patients with behaviour problems (30) included those who denied the seriousness of their illness and wanted to take their own discharge, sexually provocative behaviour, and those resentful about their dependent relationship with the staff. They found that numbers of patients referred to them for anxiety and denial were at their peak on the second day in the unit, while referrals for depression were at their maximum on the fourth day.

On the whole the psychiatrists were able to use fairly simple methods in dealing with these, psychiatrically speaking, straightforward situations. They prescribed tranquillizers; had frank discussions with the patients about the realities of their condition and future prospects, and bolstered optimism wherever possible; and they often recommended newspapers, radio, or sitting out in a chair. On one occasion one of the authors hypnotized an anxious patient over the telephone, but on the whole they were encouraging the patients, giving them an opportunity to express their anxieties, and reassuring the staff. The mortality of patients seen in consultation was one-third of that expected on the average, but no conclusions are drawn from this finding.

As with all investigations of this kind, the critic can say: That is very interesting but how does it help me treat my patient? In the second of these studies the answer is simple enough. An awareness of these factors-and that means being sensitive to the patient's feelings-can lead to simple preventive or remedial measures rather than waiting until there is a crisis in the ward. In the first paper, dealing with aetiological factors, the answer is not so clear. The kind of emotional events-rows and rejections-they found are not something a doctor can advise his patient to avoid. At best they will only be replaced by silent resentment, which may in the end be more damaging. Neither can bereavements or redundancy at work be deliberately avoided.

Rather a knowledge of antecedent events enables the doctor to reach a fuller understanding of his patient, something of the setting of the illness. Just as the heart is part of a human body, so the patient is part of a family and society, and a person cannot be understood without any reference to those others with whom he shares his life. Further, a knowledge of the background can help in assessing prognosis and planning future management. It may be possible as a result of the crisis (admission to hospital for cardiac failure) to bring about some changes in the physical and human environment. Social and psychological factors underlie all illness, and they are readily apparent to anyone who shows an interest in his patient as a person. They are also amenable to study, as was shown at a conference devoted to "Life Events and Psychosomatic Disorder" on 1-2 October organized by the Society for Psychosomatic Research at the Royal College of Physicians of London. A sensitivity to these psychological and social factors can enhance the quality of every doctor's work. And how does he begin? He listens to his patient.

1 Beaumont, W., Experience and Observations on the Gastric fuice and Physiology of Digestion, ed. A. Combe. Edinburgh, Maclachlan and Stewart, 1838

2 Perlman, L. V., Ferguson, S., Bergum, K., Isenberg, E. L., and Hammarsten, J. F., Annals of Internal Medicine, 1971, 75, 1

3 Cassem, N. H., and Hackett, T. P., Annals of Internal Medicine, 1971, $75,9$.

\section{Clarity and Confusion in Active Chronic Hepatitis}

The results of the first controlled prospective trial of corticosteroid treatment for active chronic hepatitis in Great Britain, published recently by G. C. Cook, R. Mulligan, and Sheila Sherlock, ${ }^{1}$ showed that it improved the likelihood of survival. The concentration of serum albumin rose during therapy, presumably because of an improvement in hepatic synthesis. The concentration of serum transaminase, though usually regarded as an index to hepatocellular necrosis, was found to be of no value in assessing the effectiveness of therapy, for it often fell towards normal without corticosteroids. The overall findings are in agreement with those of other recent reports, including one from the Mayo Clinic, and with the findings of the Copenhagen trial of steroid therapy for cirrhosis of the liver. ${ }^{2}$ In the Copenhagen study, perhaps one of the best controlled trials yet to be carried out in liver disease, the sole criterion for admission was a histological diagnosis of cirrhosis. Of the 334 patients finally included the only ones to show a significantly lower mortality with steroid therapy were "young females without ascites," which is the group most likely to include patients with active chronic hepatitis.

But there are still many questions to be answered, and perhaps the greatest problem of all lies in the definition of active chronic hepatitis. To the practising clinician this brings to mind the picture of a patient, often an adolescent girl, who has been jaundiced for some months. The onset has usually been insidious, though it is occasionally acute and similar to that of infectious hepatitis. On investigation the patient is often found to have clinical or laboratory evidence of disease in other systems - skin lesions, arthropathy, ulcerative colitis, thyroid disease, fibrosing alveolitis, neuropathy, renal tubular acidosis, or Sjögren's syndrome, the last two being particularly common, with an incidence in some series of up to $40 \% .^{3}$ Antinuclear factor and antibodies to smooth muscle are present in about half of the patients, but lupus erythematosus cells are not found as frequently as was initially described. A fairly consistent feature is hypergammaglobulinaemia, but the changes in standard liver function tests vary considerably. The criteria for inclusion in the Mayo clinic series included a ten-fold increase in serum transaminase and a two-fold increase in gamma globulin, but Cook and colleagues comment that if they had adopted the same criteria they would have had insufficient cases to undertake the trial.

Characteristic histological changes are also described. These include infiltration of the portal tracts by lymphocytes and plasma cells, with necrosis of the surrounding liver cells (so called "piece-meal necrosis"), together with fibrosis and distortion of lobular architecture. But confusion has arisen because the occurrence of such histological features in the absence of cirrhosis has been termed "chronic aggressive hepatitis."4 Very occasionally chronic aggressive hepatitis can resolve, but usually, as was recognized by the group of pathologists who were concerned in this classification, the lesion is associated with the clinical features of active chronic hepatitis and progresses rapidly to cirrhosis. In fact at the time of diagnosis cirrhosis is frequently already present. This was so in the $\mathbf{3 5}$ of Cook and colleagues' 44 patients in whom histological material was available for assessment. It is to be noted also that from specimens ob- 
tained by percutaneous liver biopsy it is often impossible to be certain about the absence of cirrhosis.

Since cirrhosis, then, is one of the most consistent features of this condition, both the clinical label of active chronic hepatitis and the term chronic aggressive hepatitis-as used by the pathologist in describing the pathological appearances -are as inappropriate as they are confusing. Furthermore, in active chronic hepatitis different areas of the liver may vary greatly in appearance. Indeed, this was why Cook and colleagues did not attempt to analyse the serial biopsies in their patients for the effects of the corticosteroid therapy, though the histological appearances of chronic aggressive hepatitis were considered to be of importance in deciding on the inclusion of patients in their trial.

In most series of patients with active chronic hepatitis there are some with few or no clinical signs and with minimal changes in liver function tests, though the histological appearances indicate considerable activity of the underlying process. Other cases like these may pass unrecognized until the cirrhosis has become gross. By that time the histological appearances of activity may have lessened, and if the presentation of the patient at that stage is with variceal bleeding, encephalopathy, or ascites the diagnosis usually made is one of cryptogenic cirrhosis. The term cryptogenic cirrhosis is not particularly satisfactory, for in many cases liver biopsy shows some evidence of inflammatory activity, and the same autoantibodies may be present in the serum (though at a lower frequency) as in patients with active chronic hepatitis. Indeed, Deborah Doniach and J. G. Walker ${ }^{5}$ have proposed that active chronic hepatitis and cryptogenic cirrhosis as well as primary biliary cirrhosis are all part of the spectrum of autoimmune liver disease. Though some of the clinical and histological features of primary biliary cirrhosis are distinct, recent studies have indicated some overlap with active chronic hepatitis. Certain patients with the latter condition, for instance, have some of the features of biliary cirrhosis in the liver biopsy and a considerably raised serum alkaline phosphatase level, which is more characteristic of primary biliary cirrhosis.

Disease in several systems is also found in cryptogenic cirrhosis, though less frequently than in active chronic hepatitis or primary biliary cirrhosis. Histological examination of the organs affected, including salivary gland, kidney, or lung in all three diagnostic groups, discloses varying degrees of lymphocytic infiltration like that seen in the liver. Such infiltration is suggestive of a cell-mediated immune response, and recently M. G. M. Smith and colleagues ${ }^{6}$ have shown with the leucocyte migration test (a known measure of cellular hypersensitivity) that abnormal responses can be detected in some patients with active chronic hepatitis, primary biliary cirrhosis, or cryptogenic cirrhosis. Furthermore, in those patients who had developed renal tubular acidosis or Sjögren's syndrome abnormal responses to kidney and salivary antigens could also be detected. These workers suggested that damage to the liver initially from virus, drug, toxin, or unknown agent led to release or alteration of a cell antigen, which in patients with an abnormal central immune system is followed by a cell-mediated response directed against the liver and other organs with common antigens. If the cell-mediated response were directed mainly against cell surface antigens in active chronic hepatitis and cryptogenic cirrhosis, and against bile ductule antigens in primary biliary cirrhosis, this could account for the differences in clinical picture usually observed.

Since corticosteroid therapy is effective in prolonging survival in active chronic hepatitis, and since asymptomatic cases have had florid histological changes progressing to cirrhosis, it may be unwise to stop treatment as soon as the clinical and biochemical features return to normal. For even though the disease may appear to be inactive when assessed by all possible methods (including immunological) no controlled trial has yet shown whether further treatment, perhaps by preventing a relapse, is beneficial or harmful.

The value of azathioprine, both initially in the control of the disease and as maintenance therapy, is also uncertain at present. The preliminary reports of the Mayo Clinic controlled trial indicate that it is less effective than corticosteroids. ${ }^{2}$ Perhaps this is not surprising, for the immunosuppressive activity of azathioprine is thought to be dependent on its metabolic transformation, probably in the liver, and it has been shown that the level of immunosuppressive activity attained in the serum is reduced when liver function is impaired. ${ }^{7}$ There are also some reports suggesting that patients with liver disease are unduly susceptible to the hepatotoxic effects of azathioprine and that the use of doses of more than $100 \mathrm{mg}$ per day may be followed by an increase in jaundice and the development of hepatic coma. ${ }^{8}$ Though it is possible that a combination of azathioprine and corticosteroid, each in a smaller dose, is better than either singly, this too remains to be established.

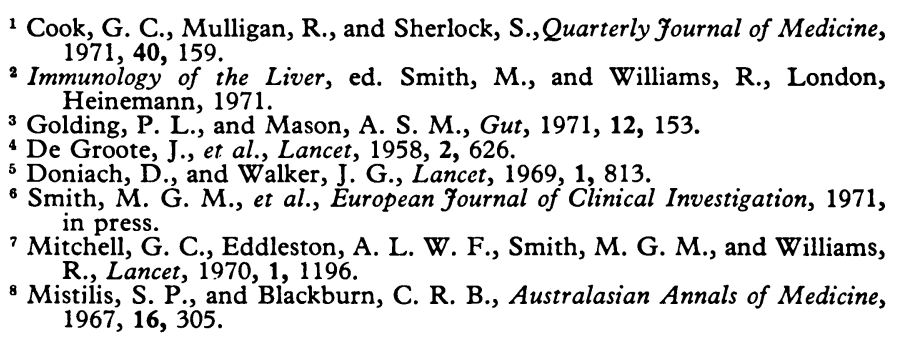

\section{Solar Flares and the Concorde}

"Any person remaining unprotected within the path of the radiation from a major flare at the distance of the Earth from the Sun will almost certainly receive a fatal dose." So writes P. M. Molton. ${ }^{1}$ The key words in this statement are "remaining" and "unprotected." Passengers in supersonic aircraft, at the height these vehicles are expected to fly, come within $3 \%$ of being full-blown astronauts, since $97 \%$ of the atmosphere is below them. But they themselves need not remain there. As to being unprotected, even a space-walking astronaut outside his ship has layer upon layer of clothing around him, not to mention a layer of gas. The occupants of an aeroplane or spaceship are shielded by the vessel wall and any objects stowed within the vessel between the wall and its occupants. All these items have some effect.

Shielding from radiation is usually reckoned in terms of grammes of matter per square centimetre of the surface being bombarded. The grammes are units of mass, not of weight-at least in space travel, where a mass of $1 \mathrm{~g}$ may weigh $6 \mathrm{~g}$ on take-off but none at all while coasting through empty space. Measured in these units, the shielding effect of the whole atmosphere is rather more than 1,000 $\mathrm{g} / \mathrm{cm}^{2}$. At the cruising height of the Concorde, somewhere between 75,000 and $80,000 \mathrm{ft}(22,900$ and $24,400 \mathrm{~m})$, atmospheric shielding is reduced to 36 and $29 \mathrm{~g} / \mathrm{cm}^{2}$ respectively. 